\title{
ANÁLISE DA RELAÇÃO PIB E DESEMPREGO NO BRASIL UMA APLICAÇÃO DO MODELO DE COINTEGRAÇÃO ASSIMÉTRICA
}

Mario Antonio Margarido ${ }^{1}$

\section{RESUMO}

Este estudo analisou a relação entre o Produto Interno Bruto do Brasil (PIB) e o número de Pessoas Desocupadas nas principais Regiōes Metropolitanas do país. Os dados são mensais e período analisado inicia-se em janeiro de 2003 e estende-se até janeiro de 2016. A fonte de dados básicos para o PIB foi o Banco Central do Brasil, e para o número de Pessoas Desocupadas foi o Instituto Brasileiro de Geografia e Estatística (IBGE). Foi utilizado o Método X12 para remover a sazonalidade de cada série. Para determinar da ordem de integração das séries foi utilizado o teste de raiz unitária Dickey-Fuller Aumentado (ADF). O relacionamento de longo prazo das séries foi analisado com o teste de Cointegração de Engle-Granger. Para analisar a questão da cointegração assimétrica foi utilizado o modelo de Enders e Siklos (2001). Os resultados mostram que as variáveis são cointegradas, sendo que, o relacionamento entre as variáveis é elástico no longo prazo, além disso, há presença de assimetria,

1 Pós-doutorado em Economia (EESP/FGV), Dr. em Economia Aplicada (Esalq/USP), Mestre em Economia de Empresas (EAESP/FGV), Economista (FEA/USP). Assessoria de Política Tributária e Econômica (APTE), Secretaria da Fazenda do Estado de São Paulo. E-mail: margaridoma@gmail.com. 
ou seja, choques negativos têm impactos mais acentuados sobre o desemprego do que choques positivos.

Palavras-chave: Cointegração assimétrica; Sazonalidade; Desemprego; Produto.

\section{ABSTRACT}

This study examined the relationship between the Gross Domestic Product of Brazil (GDP) and the number of unemployed persons in the main metropolitan areas of the country. The data are monthly and analyzed period begins in January 2003 and extends until January 2016. The source of basic data for GDP was the Central Bank of Brazil, and the number of unemployed persons was the Brazilian Institute of Geography and Statistics (IBGE). $X 12$ method was used to remove the seasonality of each series. To determine the order of integration of the series was used unit root test Dickey-Fuller $(A D F)$. The long-term relationship of the series was analyzed with the Cointegration of Engle-Granger test. To examine the issue of asymmetric co-integration model was used Enders and Siklos (2001). The results show that the variables are co-integrated, and the relationship between variables is elastic in the long run, furthermore, there is presence of asymmetry, namely, negative shocks have more severe impact on the unemployment than positive shocks.

Keywords: Asymmetric cointegration; Seasonality; Unemployment; Output.

\section{INTRODUÇÃO}

Os manuais de macroeconomia apresentam a existência de uma relação empírica entre variaçôes no desemprego em decorrência de variações no Produto Interno Bruto (PIB). Essa relação tem como base a denominada Lei de Okun. Entretanto, diversos estudos mostram que essa não é uma relação estável ao longo do tempo. Conforme KNOTEK (2007), parte desta instabilidade está associada ao estado do ciclo de negócios da economia. Mais precisamente, o relacionamento entre produto e desemprego apresenta comportamento diferenciado em função das condições de 
expansão ou contração da economia, configurando um quadro em que permeia o dinamismo das relações entre as variáveis econômicas.

Portanto, o estágio em que se encontra a economia determina a relação entre produto e desemprego. Isto indica a presença de possíveis efeitos assimétricos entre essas duas variáveis.

A identificação de assimetrias no relacionamento entre estas duas variáveis, segundo HARRIS and SILVERSTONE (2001), tornam-se muito significativas para as estratégias dos policy makers pelos seguintes motivos: 1) permite distinguir entre teorias relativas aos mercados de bens e de trabalho, qual é a mais adequada; 2) o conhecimento sobre a extensão da assimetria entre desemprego e produto é muito útil para os formuladores de política econômica no que diz respeito à consecução de políticas estruturais, como, por exemplo, reformas no mercado de trabalho, e, também, no caso de políticas de estabilização, como por exemplo, qual deveria ser a resposta mais adequada da política monetária diante de determinada conjuntura econômica. Finalmente, a presença de assimetria na relação produto e desemprego, pode gerar viés nas previsões de variáveis macroeconômicas. Daí a importância em se investigar a presença de assimetria na Lei de Okun para a economia brasileira recente.

\section{REFERENCIAL TEÓRICO: A LEI DE OKUN}

Historicamente, a relação entre PIB e desemprego foi inicialmente identificada pelo economista Arthur Okun do Council of Economic Advisers (CEA), na gestão do governo do presidente Kennedy, nos anos 60s. Essa relação passou a ser denominada de Lei de Okun. Em linhas gerais, ela tem como base os seguintes pressupostos. Para cada economia específica há uma determinada taxa "normal" de crescimento. Quando o PIB de se expande acima de sua taxa normal de crescimento, isso resulta em redução do desemprego. No entanto, é necessário observar que essa relação não é direta e pode variar em razão das características de cada economia e de seu respectivo ponto no ciclo de negócios.

Conforme apresentado em BLANCHARD (1997), matematicamente, a denominada Lei de Okun é representada pela seguinte fórmula:

$$
u_{t}-u_{t-1}=-\beta\left(g_{y t}-\overline{g_{y}}\right) \text {, }
$$

onde o termo $u_{t}-u_{t-1}$ representa o desvio do desemprego em relação a sua taxa natural. Enquanto que, $g_{y t}$ corresponde ao Produto Observado e $\overline{g_{y}}$ representa o 
Produto Potencial ${ }^{2}$. Portanto, a Lei do Okun relaciona como o crescimento no Produto determina mudanças na taxa de desemprego. Conforme preceitua essa Lei, o valor do $\beta$ estimado, também denominado de coeficiente de Okun, deve ser negativo, logo, quando o Produto aumenta, o desemprego, necessariamente, diminui.

Para capturar não somente os efeitos contemporâneos entre as duas variáveis, foi desenvolvida a versão dinâmica da Lei do Okun, a qual incorpora ao modelo variáveis defasadas ${ }^{3}$.

Dadas as dificuldades para se estimar o Produto Potencial da Economia, para o seu cálculo, geralmente, utiliza-se o denominado Filtro HP, conforme desenvolvido por HODRICK e PRESCOTT (1997). Em linhas gerais, o Filtro HP produz uma tendência suavizada da série original.

$\mathrm{Na}$ literatura sobre ciclos econômicos, há predominância na estimação do Produto Potencial de uma economia utilizando-se o Filtro HP, o qual é um modelo univariado, para se obter o Hiato do Produto. No entanto, esse não é o único procedimento disponível para se determinar o Hiato do Produto. Conforme apresentado em Brouwer (1998), além do Filtro HP univariado, pode-se estimar o Hiato do Produto, pelo método de Tendência Linear, o qual também é univariado, ou então, com o uso de modelos multivariados, como, Método Hodrick-Prescott Multivariado, Método de Componentes não Observáveis e Método de Função de Produção.

Ao se aplicar o logaritmo nos dois lados da equação acima, obtêm-se a taxa de variação do desemprego e o Hiato do Produto ${ }^{4}$, que, também, consiste na taxa de variação do Produto. Nesse caso, o valor de $\beta$ representa a elasticidade produto/ desemprego.

No entanto, ao se trabalhar com as respectivas taxas de variações, isso quer dizer que os elementos de longo prazo das séries são removidos, e, consequentemente, seus resultados somente podem ser analisados em termos de curto prazo, pois as respectivas tendências, as quais captam os elementos de longo prazo, são removidas.

Para comprovar a afirmação do parágrafo anterior foi estimado 5 o modelo apresentado por Okun. Inicialmente, dado que foi constado que as séries do PIB e

Também denominado de Produto Médio.

3 Geralmente, utiliza-se algum Critério de Informação na determinação do número correto de defasagens.

4 Lembrando que o Hiato do Produto consiste no $\ln \left(g_{y t} / \overline{g_{y}}\right)$. Quando o Hiato é maior que zero, isso implica que o Produto Observado é maior que o Produto Potencial, ocorrendo o contrário quando o Hiato é menor que zero.

5 Os resultados deste modelo não serão apresentados em função da restrição de espaço. 
do número de Desocupados apresentavam sazonalidade, utilizou-se o Método X12 para remoção das respectivas sazonalidades. A seguir, foi utilizado o Filtro HP para se estimar o Produto Potencial e o Número de Desocupados "potencial". Uma vez obtidas essas variáveis, foram calculados os respectivos hiatos. O próximo passo consistiu em determinar a ordem de integração de cada hiato utilizando-se o teste de raiz unitária $\mathrm{ADF}$. Os testes para as duas variáveis mostraram que ambas são estacionárias em nível, ou seja, integradas de ordem zero. Dado que as séries são integradas de ordem zero, isso implica na impossibilidade de se estimar um modelo de cointegração. Sendo assim, foi estimado um modelo de regressão com o hiato de cada variável. Os resultados mostram que, apesar do coeficiente estimado para o hiato do PIB ser estatisticamente significativo e ter o sinal esperado, conforme preceitua a teoria econômica, o Coeficiente de Determinação Ajustado ( $R^{2}$ ajustado) ficou em torno de $10 \%$, somente. Além disso, o resultado do Durbin-Watson foi igual a 0,39 , indicando presença de autocorrelação positiva de ordem um. Diante da presença de autocorrelação, há dois caminhos possíveis. Um deles seria a inserção de outras variáveis no modelo, no entanto, isso descaracterizaria o modelo de Okun. Diante da constatação de autocorrelação nos resíduos, a segunda alternativa, é diferenciar as variáveis. No entanto, a diferenciação não pode ser efetuada, uma vez que, ambos os hiatos são estacionários, e, ao se diferenciar variáveis estacionárias, gera-se variáveis sobre diferenciadas, que, por sua vez, produz resultados viesados.

Conforme SOUZA JÚNIOR e CAETANO (2013, p. 7), no "longo prazo, o Produto Interno Bruto (PIB) efetivo não pode crescer a taxas médias mais elevadas que as do produto potencial. Isso significa que o crescimento da economia pode ser maior ou menor do que o crescimento potencial no curto prazo, mas, na média, para períodos mais longos, esses dois indicadores devem coincidir". Portanto, com base na observação acima, dado que, no longo prazo, o produto observado tende a ser igual ao produto potencial, ao invés de se utilizar o hiato do produto, este será substituído pela variável produto em nível, sendo que, o mesmo se aplica ao número de pessoas Desocupadas para verificar se as variáveis cointegram, isto é, se realmente convergem para o equilíbrio no longo prazo.

\section{OBJETIVOS E MÉTODOS}

\subsection{Objetivos}

Dado que, no curto prazo, os mercados podem estar fora do equilíbrio, em função de ruídos nesses mercados, tais como, restrições contratuais, políticas co- 
merciais etc., a ciência econômica enfatiza a análise de longo prazo, pois, a expectativa é de que, no longo prazo, todos os mercados superem esses ruídos e convirjam para o equilíbrio.

Sendo assim, o objetivo desse estudo reside em analisar se essas variáveis cointegram, ou seja, convergem para o equilíbrio no longo prazo utilizando o teste de cointegração desenvolvido por Engle e Granger (1991).

Caso essas variáveis cointegrem, também objetiva-se verificar se o relacionamento entre essas duas variáveis é simétrico, ou seja, se choques positivos e negativos sobre o Produto têm o mesmo efeito sobre o Desemprego, ou então, se é assimétrico, isto é, choques negativos no Produto têm efeitos mais severos sobre o desemprego do que choques positivos. Conforme preceitua a literatura sobre assimetria, más notícias (badnews) têm efeitos mais relevantes do que boas notícias (goodnews).

\subsection{Material}

Foram coletadas duas séries mensais, uma relacionada ao Produto Interno Bruto (PIB) do Brasil e População Desocupada nas Regiōes Metropolitanas de Recife, Salvador, Belo Horizonte, Rio de Janeiro, São Paulo e Porto Alegre, sendo o período de referência igual a 30 dias. O período analisado inicia-se em janeiro de 2003 e termina em janeiro de 2016, totalizando 157 observações.

A série do PIB está em R \$ milhões, enquanto que, a População Desocupada das principais Regiões Metropolitanas está em mil pessoas. A série do PIB do Brasil foi estimada pelo Banco Central do Brasil, e foi obtida no site do Ipeadata. A série da População Desocupada tem como base o Instituto Brasileiro de Geografia e Estatística (IBGE), e foi obtida no site do Sistema IBGE de Recuperação Automática (Sidra). Conforme consta no site do IBGE, o conceito de População Desocupada envolve aquelas pessoas que não tinham trabalho, num determinado período de referência, mas estavam dispostas a trabalhar, e que, para isso, tomaram alguma providência efetiva (consultando pessoas, jornais etc.).

A análise visual dos respectivos correlogramas ${ }^{6}$, em nível e na diferença, revelou que as duas séries apresentam sazonalidade. Sendo assim, foi utilizado o Método X12 para remover a sazonalidade de cada série.

Após as séries serem dessazonalizadas, aplicou-se o operador logarítmico. Sendo assim, LDESOCUPADOS_SA representa o logaritmo da População Desocupada

6 Os respectivos correlogramas tanto em nível quando na diferença não serão aqui apresentados. 
dessazonalizada nas principais Regiões Metropolitanas, por sua vez, LPIB_SA corresponde ao logaritmo do PIB do Brasil, também, dessazonalizado.

\subsection{Métodos}

Basicamente, uma série de tempo pode ser desagregada em quatro componentes: ciclo, tendência, sazonalidade e componente aleatório. Matematicamente, uma série de tempo pode ser multiplicativa ou aditiva, conforme apresentado a seguir, respectivamente:

$$
\begin{aligned}
& \widehat{Y m}_{t}=\widehat{T}_{t} * \widehat{S}_{t} * \widehat{C}_{t} * \widehat{I}_{t} \\
& \widehat{Y a}_{t}=\widehat{T}_{t}+\widehat{S}_{t}+\widehat{C}_{t}+\widehat{I}_{t}
\end{aligned}
$$

${\widehat{Y m_{t}}}_{t}$ é série de tempo classificada como Multiplicativa; $\widehat{Y a}_{t}$ é a série de tempo classificada como Aditiva; $T$, Tendência; $S$, Sazonalidade; $C$, Ciclo; e $I$, Componente Aleatório, também denominado de Componente Irregular.

De acordo com FREDO e MARGARIDO (2008, p. 372), a "tendência capta elementos de longo prazo relacionados com a série de tempo; pode ser determinística, ou seja, pode ser uma função matemática, ou então estocástica ${ }^{7}$, isto é, resultado de um processo aleatório".

O componente sazonal capta os padrões regulares da série de tempo, tais como mudanças de temperatura, índice pluviométrico, safra ou entressafra de produtos agropecuários, vendas da indústria, vendas do varejo, entre outros.

Os ciclos são caracterizados por longas ondas, mais ou menos regulares, em torno de uma linha de tendência. $\mathrm{O}$ interesse pelos ciclos está diretamente relacionado com seus pontos de mudanças, também denominados de pontos de inflexão ${ }^{8}$, duração, frequência, etc.".

Detalhes sobre o método X12 podem ser encontrados em Yaffee e McGee (2000).

No cálculo do Coeficiente de Amplitude Sazonal (CA) de cada série foi utilizado o trabalho de Freitas et al. (1998). Esse coeficiente tem como base a seguinte fórmula:

7 Neste caso, a tendência estocástica, em economia, mostra alteraçôes no nível da série de tempo, as quais podem ser resultado de mudanças sociais, tecnológicas, condiçóes de mercado, meio ambiente, entre outros.

8 Em economia, o interesse em conhecer adequadamente este componente da série de tempo está relacionado com os ciclos econômicos, visando determinar períodos de prosperidade, recessão, entre outros. 
-• Economia Brasileira em Debate

$$
C A \%=\frac{(\text { Índice Máximo }- \text { Índice Mínimo })}{(\text { Índice Máximo }+ \text { Índice Mínimo })} * 2 * 100
$$

Para a determinação da ordem de integração de cada variável, foi utilizado o teste de raiz unitária Dickey-Fuller Aumentado (ADF), conforme apresentado em DICKEY e FULLER (1979 e 1981). No entanto, antes da realização do teste de raiz unitária é necessário determinar o número de defasagens a ser utilizada em cada fase do teste de raiz unitária. A correta determinação do número de defasagens é de suma importância, pois é necessário garantir que os resíduos das autorregressões dos testes de raiz unitárias sejam não correlacionados, de forma a garantir resultados não viesados dos respectivos testes. Para a determinação do número de defasagens foi utilizado o Critério de Informação de Akaike Corrigido (AICC). O Critério de Akaike Corrigido (AICC), conforme definido por HURVICH e TSAI (1989), é definido como:

$$
A I C C=\log (|\Sigma|)+\frac{2 r}{(T-r / K)}
$$

sendo que, $r$ corresponde ao número de parâmetros estimados, $\Sigma$ denota a estimativa de máxima verossimilhança da matriz de variância-covariância $(\Sigma)$, e $k$ representa a dimensão da série temporal. O AICC se caracteriza por ser assintoticamente eficiente tanto para os modelos de regressão, quanto para o caso de modelos de séries temporais, e, além disso, tem melhores propriedades estatísticas do que o Critério de Informação de Akaike (AIC).

Visando identificar a possível relação de longo prazo entre as variáveis, foi utilizado o teste de cointegração desenvolvido por ENGLE e GRANGER (1991). Basicamente, este teste é conduzido em duas etapas. Dado que as variáveis envolvidas tenham a mesma ordem de integração, deve-se estimar uma equação de regressão com as variáveis em nível, conforme apresentado em (5). Esta equação também é denominada de equação de cointegração:

$$
\mathrm{y}_{t}=\alpha+\beta x_{t}+u_{t}
$$

onde $\alpha$ corresponde ao intercepto da regressão e $u_{t}$ são os resíduos da regressão.

Na verdade, a equação de cointegração apresentada em (5), nada mais é do que a equação de regressão tradicional com as variáveis em nível. Uma vez estimada esta regressão com as variáveis em nível e dado que ambas as variáveis são integradas de ordem 1, o próximo passo consiste em “capturar" os resíduos $\left(u_{t}\right)$ da 
equação de cointegração, e, posteriormente testar se os resíduos são estacionários $I(0)$ ou não são estacionários, isto é $I(1)$. Os valores críticos para os testes de raiz unitária sobre os resíduos da equação de cointegração podem ser encontrados em ENGLE e GRANGER (1991), PHILLIPS e OULIARIS (1990) e MACKINNON (1991).

Portanto, dado que o teste de cointegração envolve a realização de teste de raiz unitária do tipo Dickey-Fuller Aumentado (ADF) sobre os resíduos da equação de cointegração, este teste deve ser efetuado sobre a seguinte equação de resíduos:

$$
\nabla u_{t}=\rho u_{t-1}+\sum_{i=1}^{p} \gamma_{i} \nabla u_{t-i}+e_{t}
$$

Neste caso, o termo $\nabla u_{t}$ representa a primeira diferença da variável $u_{t}$ enquanto que, $\nabla u_{t-i}$ é a primeira diferença defasada da variável $u_{t}$, sendo que, o número total de defasagens corresponde a $i$ períodos. Quando o termo $\gamma_{i}$ é igual a zero para todos os is, ao invés do teste Dickey-Fuller Aumentado (ADF), tem-se o teste Dickey-Fuller (DF).

Tendo como base os resíduos da equação de cointegração, o procedimento de verificação da presença ou não de raiz unitária testa a hipótese nula de que os resíduos têm raiz unitária $(\rho=0)$ contra a hipótese alternativa de que os resíduos são estacionários $(\rho \neq 0)$, ou seja, testa a hipótese nula de que não há cointegração versus a hipótese alternativa de que existe cointegração entre as variáveis $y_{t}$ e $x_{t}$.

Detectada a relação de cointegração entre as variáveis, o próximo passo consiste na inclusão do modelo de correção de erro. ENGLE e GRANGER (1991) mostraram que se $y_{t}$ e $x_{t}$ são ambas integradas de ordem um $(I(1))$ e são também cointegradas, então, existe uma representação de modelo de correção de erro da seguinte forma:

$$
\nabla y_{t}=\omega_{0}+\omega_{1} \nabla x_{t}-\gamma\left(y_{t-1}-\alpha x_{t-1}\right)+\varepsilon_{t},
$$

onde $\nabla y_{t}$ e $\nabla x_{t}$ representam as variáveis $y_{t}$ e $x_{t}$, respectivamente. Dado que representam as diferenças das variáveis esses dois elementos captam os efeitos de curto prazo do modelo de correção de erro. O parâmetro $\gamma$ mostra a velocidade pela qual os desvios em relação à condição de equilíbrio são corrigidos em cada período de tempo e também é denominado de termo de correção de erro. Portanto, quanto maior o valor estimado de $\gamma$, mais rapidamente os desvios são corrigidos. Por outro lado, quanto menor o seu valor, mais tempo leva para o sistema voltar a uma nova posição de equilíbrio. Finalmente, o termo $\left(y_{t-1}-\alpha x_{t-1}\right)$ representa os resíduos da 
equação de cointegração, porém, defasados de um período. Lembrando que $z_{t}=y_{t}-\alpha x_{t}$, ou seja, $z_{t}$ corresponde aos resíduos da equação de cointegração. Defasando-se essa equação em um período tem-se $z_{t-1}=y_{t-1}-\alpha x_{t-1}$, sendo que o lado direito dessa última expressão corresponde aos resíduos defasados de um período, conforme apresentado em (5).

Também, será utilizado o teste de cointegração assimétrica conforme apresentado em ENDERS e SIKLOS (2001). O teste de cointegração assimétrica é uma derivação do teste de Engle-Granger, pois os procedimentos iniciais são idênticos aos do teste de Engle-Granger. A distinção é que no caso do teste de assimetria, os resíduos defasados de um período são divididos em duas partes. Segundo HARRIS e SOLLIS (2003) uma especificação alternativa para a equação (8), a qual é denominada de momentum threshold autoregressive model, pode ser representada como:

$$
\nabla \hat{u}_{t-1}=I_{t} \rho_{1} \hat{u}_{t-1}+\left(1-I_{t}\right) \rho_{2} \hat{u}_{t-1}+V_{t}^{*} \sim\left(0, \sigma^{2}\right),
$$

onde $I_{t}=1$ se $\hat{u}_{t-1} \geq 0$ e $I_{t}=0$ se $\hat{u}_{t-1}<0$. Em relação a este teste, a hipótese nula afirma que as variáveis não são cointegradas, enquanto que, na hipótese alternativa elas são cointegradas, porém, com assimetria. Matematicamente, a hipótese nula é $\left(\rho_{1}+\rho_{2}=0\right)$ e pode-se utilizar a convencional estatística $F$.

\section{ANÁLISE DE RESULTADOS}

Com base no Método da Decomposição X12, foram calculados os Índices Médios Sazonais para as duas séries. No caso da variável PIB do Brasil, o maior Índice Sazonal Médio ocorre em novembro com valor igual a 103,2, enquanto que, o menor, ocorre em fevereiro, com valor igual a 93,7. Aparentemente, esses resultados são robustos, dado que, a produção industrial tem seu pico sazonal em outubro, em decorrência da produção dos produtos para serem entregues ao varejo em novembro e posterior comercialização em dezembro, período de festas de final de ano. Por outro lado, o menor índice em fevereiro se justifica pelo fato de que esse mês tem menor número de dias úteis comparativamente aos demais meses do ano. Além disso, esse efeito de redução do PIB é magnificado pelo carnaval, que, na maioria das vezes, ocorre em fevereiro, reduzindo ainda mais o número de dias úteis, impactando não somente a indústria, como o próprio varejo. $\mathrm{O}$ coeficiente de amplitude sazonal do PIB do Brasil é igual a 9,64\%. Esse resultado indica que essa variável apresenta sazonalidade, porém, não muito intensa. 
Quanto a variável Pessoal Desocupado das principais regióes metropolitanas, o maior Índice Sazonal Médio ocorre em março, sendo igual a 105,7 e o menor em dezembro com magnitude igual a 85,2. Em relação a essa variável, os resultados também são sustentáveis, pois o mês dezembro é aquele que demanda maior quantidade de mão-de-obra pelo setor varejista em decorrência das festas de final de ano, melhor mês de vendas para esse segmento. O maior Índice Sazonal Médio em março, possivelmente, está relacionado ao fato de que é final de verão e o comércio diminui o número de vagas de trabalhadores temporários. A variável pessoal desocupado apresenta coeficiente de amplitude sazonal muito mais pronunciado comparativamente ao PIB, com magnitude igual a $21,47 \%$.

A justificativa metodológica para a remoção da sazonalidade das duas séries reside no fato de que o teste de raiz unitária $\mathrm{ADF}$ gera resultados viesados quando a séries apresenta sazonalidade, sendo que, o mesmo se aplica em relação ao teste de cointegração de Engle-Granger. Ao se trabalhar com modelos de séries temporais, o primeiro passo consiste em determinar a ordem de integração das variáveis utilizadas via algum teste de raiz unitária. No entanto, antes da determinação da ordem de integração com a utilização de testes de raiz unitária, torna-se necessário determinar o número de defasagens tanto para as variáveis consideradas em nível quanto nas diferenças, caso seja necessário diferenciar as variáveis para se obter suas respectivas estacionariedades. Nesse estudo, para a determinação do número de defasagens foi utilizado o Critério de Informação de Akaike Corrigido (AICC). Os resultados do AICC para as variáveis em nível mostram que, nos casos das variáveis LDESOCUPADOS_SA e LPIB_SA, é necessária a inserção de três defasagens para cada uma delas (Tabela 1).

Tabela 1 Critério de Informação de Akaike Corrigido (AICC), variáveis em nível, LDESOCUPADOS_SA e LPIB_SA, janeiro de 2003 a janeiro de 2016.

\begin{tabular}{|l|c|}
\hline \multicolumn{1}{|c|}{ Variáveis em nível } & Número de defasagens \\
\hline LDESOCUPADOS_SA & 1 \\
\hline LPIB_SA & 1 \\
\hline
\end{tabular}

Fonte: Elaborada pelos autores a partir de dados básicos do IBGE e Bacen.

Resultados dos testes de raiz para os três modelos, quais sejam, com tendência e constante, somente com constante e sem tendência e sem constante, mostram que em todos os casos a hipótese nula de raiz unitária não pode ser rejeitada, 
•• Economia Brasileira em Debate

pois, todos os p-values não são estatisticamente significativos, uma vez que se encontram acima de $10 \%$ de probabilidade. Portanto, a probabilidade de se cometer o Erro Tipo I, rejeitar a hipótese nula e ela ser verdadeira é muito elevada, logo, não se rejeita a hipótese de raiz unitária para as duas variáveis (Tabela 2).

Tabela 2 Resultados dos Testes de Raiz Unitária ADF, variáveis em nível, LDESOCUPADOS_SA e LPIB_SA, janeiro de 2003 a janeiro de 2016.

\begin{tabular}{|l|c|c|c|c|c|c|}
\hline \multicolumn{1}{|c|}{ Variável } & $\tau_{\tau}{ }^{1}$ & $\operatorname{Pr}<\tau_{\tau}$ & $\tau_{\mu}{ }^{2}$ & $\operatorname{Pr}<\tau_{\mu}$ & $\tau^{3}$ & $\operatorname{Pr}<\tau$ \\
\hline LDESOCUPADOS_SA & 0.24 & 0.9981 & -1.26 & 0.6475 & -1.11 & 0.2414 \\
\hline LPIB_SA & -0.79 & 0.9636 & -1.56 & 0.4994 & 7.31 & 0.9999 \\
\hline
\end{tabular}

${ }^{1}$ Modelo com tendência e constante; ${ }^{2}$ Modelo sem tendência, porém com constante; ${ }^{3}$ Modelo sem tendência e sem constante.

Fonte: Elaborada pelos autores a partir de dados básicos do IBGE e Bacen.

A seguir, também com a utilização do AICC, foram determinados os respectivos números de defasagens, porém, com as variáveis diferenciadas. Nos casos das duas variáveis também é necessária a inclusão de uma defasagem (Tabela 3).

Tabela 3 Critério de Informação de Akaike Corrigido (AICC), variáveis diferenciadas, LDESOCUPADOS_SA e LPIB_SA, janeiro de 2003 a janeiro de 2016.

\begin{tabular}{|l|c|}
\hline \multicolumn{1}{|c|}{ Variáveis em nível } & Número de defasagens \\
\hline LDESOCUPADOS_SA & 1 \\
\hline LPIB_SA & 1 \\
\hline
\end{tabular}

Fonte: Elaborada pelos autores a partir de dados básicos do IBGE e Bacen.

Uma vez determinado o número de defasagens para cada variável diferenciada, o próximo passo foi à realização dos respectivos testes de raiz unitária. Os resultados mostram que para todas as variáveis e para todos os modelos, a probabilidade de se cometer o Erro Tipo I em cada caso é muito pequena, ou seja, menor que 1\%. Logo, rejeita-se a hipótese nula de raiz unitária para cada variável e para cada modelo (Tabela 4). Sendo assim, dada a necessidade de se aplicar uma diferença de ordem um para cada variável, isso implica que todas as variáveis são integradas de ordem um, ou seja, são diferenças estacionárias, uma vez que, a aplicação da diferenciação em cada uma torna-as estacionárias. 
Tabela 4 Resultados dos Testes de Raiz Unitária ADF, variáveis diferenciadas, LDESOCUPADOS_SA e LPIB_SA, janeiro de 2003 a janeiro de 2016.

\begin{tabular}{|l|c|c|c|c|c|c|}
\hline \multicolumn{1}{|c|}{ Variável } & $\tau_{\tau}{ }^{1}$ & $\operatorname{Pr}<\tau_{\tau}$ & $\tau_{\mu}{ }^{2}$ & $\operatorname{Pr}<\tau_{\mu}$ & $\tau^{3}$ & \multicolumn{1}{c|}{$\operatorname{Pr}<\tau$} \\
\hline LDESOCUPADOS_SA & $-8.35^{*}$ & $<.0001$ & $-8.22^{*}$ & $<.0001$ & $-8.16^{*}$ & $<.0001$ \\
\hline LPIB_SA & $-12.25^{*}$ & $<.0001$ & $-11.98^{*}$ & $<.0001$ & $-7.92^{*}$ & $<.0001$ \\
\hline
\end{tabular}

${ }^{1}$ Modelo com tendência e constante; ${ }^{2}$ Modelo sem tendência, porém com constante; ${ }^{3}$ Modelo sem tendência e sem constante. ${ }^{*}$ Significativo a $1 \%$.

Fonte: Elaborada pelos autores a partir de dados básicos do IBGE e Bacen.

Para analisar a relação de longo prazo entre as variáveis, torna-se necessária a utilização do teste de cointegração. Neste estudo será utilizado o teste de cointegração de Engle-Granger. A primeira etapa desse teste consiste em estimar uma regressão com as variáveis em nível. Essa regressão também é denominada de equação de cointegração. Os resultados da equação de cointegração se encontram na Tabela 5.

Tabela 5 Equação de cointegração, LDESOCUPADOS_SA e LPIB_SA, janeiro de 2003 a janeiro de 2016.

\begin{tabular}{|l|c|c|c|c|}
\hline \multicolumn{1}{|c|}{ Variável } & $\begin{array}{c}\text { Estimativa } \\
\text { do parâmetro }\end{array}$ & $\begin{array}{c}\text { Erro-padrão } \\
\text { da estimativa }\end{array}$ & $\begin{array}{c}\text { Valor } \\
\text { do teste } t\end{array}$ & $\begin{array}{c}p \text {-value } \\
\text { do teste } t\end{array}$ \\
\hline Intercepto & 32.65880 & 1.39083 & $23.48^{*}$ & $<.0001$ \\
\hline Tendência & 0.01400 & 0.00105 & $13.28^{*}$ & $<.0001$ \\
\hline LPIB_SA & -2.09293 & 0.11744 & $-17.82^{*}$ & $<.0001$ \\
\hline
\end{tabular}

* Significativo em nível de $1 \%$.

Fonte: Elaborada pelos autores a partir de dados básicos do IBGE e Bacen.

Em relação à equação de cointegração, é necessário enfatizar que os valores das estimativas dos parâmetros não têm significado econômico, uma vez que as variáveis são integradas de ordem um, sendo assim, não podem ser utilizadas na análise do relacionamento entre as variáveis. A equação de cointegração é utilizada apenas para se "capturar" seus resíduos, pois, a segunda etapa do teste de cointegração de Engle-Granger consiste em utilizar os resíduos da equação de cointegração e aplicar o teste de raiz unitária sobre os resíduos da equação de cointegração. No entanto, antes da execução do teste de raiz unitária, é necessário determinar o número de defasagens que será utilizado nesse teste. Novamente, para tal tarefa, foi 
utilizado o critério de informação AICC. Os resultados do AICC apontam para a necessidade da inclusão de apenas uma defasagem na execução do teste ADF.

Quanto aos resultados do teste de raiz unitária ADF, observa-se que para os três modelos, a hipótese nula de que os resíduos possuem raiz unitária pode ser rejeitada para todos eles. Logo, não se rejeita a hipótese alternativa de que os resíduos da equação de cointegração são estacionários (Tabela 6). Em outras palavras, apesar de todas as variáveis serem integradas de ordem um, a combinação linear entre as duas variáveis produz um relacionamento estacionário entre LDESOCUPADOS_SA e LPIB_SA. Portanto, essas variáveis cointegram, ou seja, convergem para o equilíbrio no longo prazo.

Tabela 6 Resultados dos Testes de Raiz Unitária ADF, resíduos da equação de cointegração, janeiro de 2003 a janeiro de 2016.

\begin{tabular}{|c|c|c|c|c|c|c|}
\hline \multicolumn{1}{|c|}{ Variável } & $\tau_{\tau}^{1}$ & $\operatorname{Pr}<\tau_{\tau}$ & $\tau_{\mu}^{2}$ & $\operatorname{Pr}<\tau_{\mu}$ & $\tau^{3}$ & $\operatorname{Pr}<\tau$ \\
\hline Residuos & $-4.24^{*}$ & 0.0049 & $-4.25^{*}$ & 0.0008 & $-4.26^{*}$ & $<.0001$ \\
\hline
\end{tabular}

${ }^{1}$ Modelo com tendência e constante; ${ }^{2}$ Modelo sem tendência, porém com constante; ${ }^{3}$ Modelo sem tendência e sem constante. ${ }^{*}$ Significativo em nível de $1 \%$.

Fonte: Elaborada pelos autores a partir de dados básicos do IBGE e Bacen.

Dado que as variáveis cointegram, então, os parâmetros estimados na equação de cointegração não são espúrios, ou seja, seus resultados são válidos tanto em termos estatísticos quanto econômicos. Sendo assim, o coeficiente estimado associado a variável $L P I B \_S A$ representa a elasticidade de longo prazo. Portanto, um choque com magnitude igual a $1 \%$ no PIB_SA induz variação igual a $-2,09 \%$ no longo prazo. Em outras palavras, um choque de $1 \%$ em PIB_SA reduz o número de pessoas desocupadas em 2,09\% no longo prazo, configurando uma relação elástica. Também, é necessário realçar que o sinal do coeficiente está condizente com o que preceitua a teoria econômica.

O fato das variáveis cointegrarem, implica na existência de um Modelo de Correção de Erro (MCE). Conforme Banerjee (1993, p. 139), o modelo de correção de erro torna-se importante por permitir a ligação entre aspectos relacionados à dinâmica de curto prazo com os de longo prazo. Assim, os "mecanismos de correção de erro pretendem fornecer um caminho para combinar as vantagens de se modelar tanto em nível quanto nas diferenças. Em um modelo de correção de erro tanto a dinâmica do processo de ajustamento de curto prazo, (variaçôes) quanto de longo prazo (níveis), são modelados simultaneamente". 
Em relação ao curto prazo, os resultados do Modelo de Correção de Erro mostram que o coeficiente estimado associado à $D L P I B \_S A$ não é estatisticamente significativo. Economicamente, isso quer dizer que choques em DLPIB_SA não impactam no comportamento de DLDESOCUPADOS_SA no curto prazo, configurando um relacionamento perfeitamente inelástico. Aparentemente, esse resultado é robusto, pois variações no PIB não afeta o nível de pessoas desocupadas no curto prazo. Possivelmente, essa inelasticidade, reside no fato de que, no curto prazo, ao invés de contratar mais pessoas, os empresários tendem a pagar horas extras para os trabalhadores já empregados (Tabela 7).

Finalmente, o sinal do termo de correção de erro está condizente com o que preceitua a teoria econométrica, e indica que os desequilíbrios no número de pessoas desocupadas são corrigidos a uma taxa de 15,72\% em cada período (Tabela 7). Em outras palavras, os desvios são corrigidos em torno de seis meses e meio. Portanto, com base nesse resultado, pode-se inferir que o longo prazo corresponde a aproximadamente seis meses e meio.

Com base nos resultados da equação de cointegração e no termo de correção de erro, pode-se inferir que variação de $1 \%$ no PIB induz variação de $-2,09 \%$ no número de pessoas desocupadas para o período correspondente a seis meses e meio (Tabelas 5 e 7 ).

Tabela 7 Resultados do Modelo de Correção de Erro, janeiro de 2003 a janeiro de 2016.

\begin{tabular}{|l|c|c|c|c|}
\hline \multicolumn{1}{|c|}{ Variável } & $\begin{array}{c}\text { Estimativa } \\
\text { do Parâmetro }\end{array}$ & $\begin{array}{c}\text { Erro-Padrão } \\
\text { da Estimativa }\end{array}$ & $\begin{array}{c}\text { Valor } \\
\text { do Teste } t\end{array}$ & P-Value $t$ \\
\hline DLPIB_SA ${ }^{1}$ & -0.11890 & 0.11452 & -1.04 & 0.3008 \\
\hline Residuo $^{2}$ & -0.15723 & 0.03952 & $-3.98^{*}$ & 0.0001 \\
\hline
\end{tabular}

${ }^{1} D L P I B \_S A=$ Diferença do Logaritmo do PIB dessazonalizado 2 . Resíduos da equação de cointegração defasados de um período ou termo de correção de erro* Significativo em nível de $1 \%$. Fonte: Elaborada pelos autores a partir de dados básicos do IBGE e Bacen.

Dado que as variáveis são cointegradas, o próximo passo residiu em verificar se essa cointegração é assimétrica. Sendo assim, o teste de raiz unitária foi novamente aplicado, porém, dessa vez, os resíduos foram divididos em dois componentes, onde o threshold, ou valor limiar, para se determinar a divisão dos resíduos, foi obtido através da imposição de restrições sobre os resíduos, sendo que, essas restrições tomam a forma de variáveis dummies conforme apresentado em ENDERS e 
SIKLOS (2001). Caso os resíduos sejam menores ou iguais a zero, a dummy assume valor igual à unidade. No entanto, se os resíduos forem positivos, a dummy assume valor igual à zero.

Os resultados do Momentum Threshold Autoregressive Model em relação aos resíduos decompostos mostram que a hipótese nula de não cointegração assimétrica $\left(\rho_{1}+\rho_{2}=0\right)$ pode ser rejeitada, uma vez que, a probabilidade de se cometer o Erro Tipo I, ou seja, rejeitar a hipótese nula e ela ser verdadeira é inferior a 1\%, sendo assim, rejeita-se a hipótese nula e não se rejeita a hipótese alternativa (Tabela 8). Portanto, pode-se concluir que os efeitos dos resíduos decompostos são assimétricos sobre o comportamento da variável número de pessoas desocupadas.

Ainda, em relação aos resíduos decompostos, os resultados mostram que os resíduos negativos são estatisticamente significativos ao nível de significância de $1 \%$, sendo que, o mesmo não ocorre com os resíduos positivos, os quais apresentam P-valor acima de $10 \%$ (Tabela 8). Esse resultado confirma que choques negativos têm maior impacto sobre o número de pessoas desocupadas do que choques positivos, reforçando, dessa forma, o resultado do teste de assimetria.

Tabela 8 Resultados do Momentum Threshold Autoregressive Model, resíduos decompostos do Termo de Correção de Erro, janeiro de 2003 a janeiro de 2016.

\begin{tabular}{|l|c|c|c|c|}
\hline \multicolumn{1}{|c|}{ Variável } & $\begin{array}{c}\text { Estimativa } \\
\text { do Parâmetro }\end{array}$ & $\begin{array}{c}\text { Erro-Padrão } \\
\text { da Estimativa }\end{array}$ & $\begin{array}{c}\text { Valor } \\
\text { do Teste } t\end{array}$ & P-Value $t$ \\
\hline DLPIB_SA & -0.05333 & 0.12233 & -0.44 & 0.6635 \\
\hline Resíduos $\leq 0$ & -0.21120 & 0.05358 & -3.94 & 0.0001 \\
\hline Resíduos $>0$ & -0.09219 & 0.05890 & -1.57 & 0.1196 \\
\hline Fonte & Graus de Liberdade & Quadrado Médio & $\begin{array}{c}\text { Valor do } \\
\text { Teste } F\end{array}$ & P-Value F \\
\hline Numerador & 1 & 0.01005 & 14.72 & 0.0002 \\
\hline Denominador & 153 & 0.00068294 & & \\
\hline
\end{tabular}

Fonte: Elaborada pelos autores a partir de dados básicos do IBGE e Bacen.

Os resultados acima estão em linha com aqueles encontrados por SchalkandUntiedt (2000), conforme citado por Döpke (2001), uma vez que, estes dois autores concluíram que a cointegração é assimétrica, sendo que, o coeficiente para o período negativo é superior em termos absolutos quando comparado com o coeficiente 
da fase expansiva, resultado este semelhante aqui encontrado, pois, em módulo, os resíduos negativos apresentam coeficiente superior aos resíduos positivos (Tabela 8). Portanto, a partir deste resultado, pode-se inferir que os efeitos de variações no produto sobre o número de desocupados dependem dos pontos aonde a economia se encontra no respectivo ciclo de negócios.

\section{CONCLUSÕES}

Os resultados deste estudo mostram que as variáveis PIB do Brasil e Pessoal Desocupado das principais regiões metropolitanas do país cointegram, ou seja, convergem para o equilíbrio no longo prazo. Em termos quantitativos, os resultados mostram que a cada variação de $1 \%$ no PIB, o número de desocupados se retrai em $2 \%$, configurando uma relação elástica no longo prazo. No curto prazo, desequilíbrios no número de pessoas desocupadas são corrigidos em torno de $15 \%$ ao mês. Portanto, são necessários 6,5 meses para a completa correção desses desequilíbrios. Possivelmente, essa defasagem de seis meses entre variaçōes no PIB e a resposta da variável número de pessoas desocupadas esteja relacionada a vários fatores, tanto de curto quanto de longo prazo.

Ainda, de acordo com os resultados obtidos pelo modelo, no curto prazo, variaçôes no PIB não determinam variações no número de pessoas desocupadas, configurando uma relação infinitamente inelástica. A justificativa para tal resultado pode ser encontrado na função de produção da microeconomia. Diante de um choque positivo, não antecipado, a tendência dos empresários consiste, inicialmente, em pagar mais horas extras para quem já está empregado, ao invés de realizar novas contratações, sendo assim, dessa forma, o número de desocupados não tende a diminuir, pelo menos, no curto prazo. No entanto, se os empresários percebem que o aumento do produto é sustentável, ou seja, se suas expectativas são positivas em relação ao futuro, eles expandem os investimentos em capital e trabalho, e, nesse caso, o aumento do produto pode induzir a queda do número de pessoas desocupadas.

Também, os resultados mostraram que choques negativos têm impactos mais significativos sobre o comportamento da variável pessoal desocupado do que choques positivos na economia, configurando uma situação em que prevalece a assimetria, resultado este condizentes com diversos estudos internacionais. A presença de assimetria indica que o estado da economia (expansão ou contração) determina diferentes comportamentos da variável número de pessoas desocupadas. 
É necessário observar que o presente estudo mostra como variações no PIB determinam variações no desemprego, no entanto, não informa em qual setor da economia a redução do desemprego será mais acentuada, pois o PIB é o agregado de vários setores econômicos. Desta forma, seria interessante, em estudos futuros, utilizar variáveis desagregadas, ou setoriais, para aferir qual segmento é mais relevante em termos de contribuir para a redução do desemprego no Brasil.

Para finalizar, em posteriores estudos, também, fosse interessante adicionar outras variáveis ao modelo que podem impactar a relação entre PIB e pessoal desocupado, como, por exemplo, a taxa de câmbio e a inflação, uma vez que, ambas as variáveis inserem as expectativas dos agentes econômicos em relação ao futuro, e, consequentemente, sobre o nível de investimento no país no longo prazo.

\section{REFERÊNCIAS}

BANERJEE, Anindya et al. Co-integration, error-correction, and the econometric analysis of non-stationary data. New York: Oxford University Press, 1993. 329p. (Advanced Texts in Econometrics).

BLANCHARD, Oliver. Macroeconomis. Estados Unidos: Prentice Hall, 1997. 623p.

BROUWER, Gordon de. Estimating Output Gaps. Reserve Bank of Australia: Economic Research Department, Research Discussion Paper, 9809, August, 1998. 32p.

DICKEY, David A.; FULLER, Wayne A. Likelihood ratio statistics for autoregressive time series with a unit root. Econometrica, v. 49, n. 4, p. 1057-1072, jul., 1981.

DICKEY, David A.; FULLER, Wayne A. Distribution of the estimators for autoregressive time series with unit root. Journal of The American Statistical Association, v. 74, n. 366, p. 427-431, jun. 1979.

DÖPKE, J. The "Employment Intensity" of Growth in Europe. Working Papers/Kiel Institute of World Economics. (RePEc: wop: kieliw: 1021), jan./2001. Disponível em:<http://netec.mcc.ac.uk/wopec/data/papers//wopkieliw1021.html.

ENDERS, W. and SIKLOS, P. L. Cointegration and threshold adjustment. Journal of Business and Economics Statistics, v. 19, p. 166-177. 2001.

ENGLE, Robert F.; GRANGER, C.W.J. Long-run economic relationship: readings in cointegration. New York: Oxford University Press, 1991. 301p.

FREDO, Carlos Eduardo e MARGARIDO, Mario Antonio. Modelando a Sazonalidade e o Processo Gerador da Série de Tempo do Emprego Rural no Estado de São Paulo. Revista de Economia e Agronegócio, v. 6, n. 3, p. 367-394. 2008.

FREITAS, S.M. de; FERREIRA, C.R.R.P. T.; BARBOSA, M.Z. Oportunidades e entraves à expansão de dendeicultura brasileira. Agricultura em São Paulo, São Paulo, v. 45 , t. 2, p. 1-16, abr. 1998 . 
HARRIS, Richard I.D.; SOLLIS, Robert. Applied time series modeling and forecasting. England: John Wiley \& Sons, 2003. 302p.

HARRIS, Richard and SILVERSTONE, Brian. Testing for asymmetry in Okun's law: A cross-country comparison. Economics Bulletin, v. 5, n. 2, p. 1-13. 2001.

HODRICK, Robert J. and PRESCOTT, Edward C. Postwar U.S. Business Cycles: An Empirical Investigation. Journal of Money, Credit and Banking, v. 29, n. 1, february, p. 1-16. 1997.

HURVICH, C.F. and TSAI, C.L. Regression and time series model selection in small samples. Biometrika, 76 (2), 297-307, 1989.

KNOTEK, Edward S. II. How Useful Is Okun's Law? Federal Reserve Bank of Kansas City Economic Review, Fourth Quarter 2007, p. 73-103, www.kc.frb.org/publicat/ econrev/PDF/4q07Knotek.pdf.

MACKINNON, James G. Critical values for cointegration tests. In: ENGLE, R. F.; GRANGER, W. J. Long-run economic relationships: readings in cointegration. New York: Oxford University Press, 1991. p. 267-276.

PHILLIPS, Peter C.B.; OULIARIS, S. Asymptotic properties of residual based tests for cointegration. Econometrica, v. 58, n. 1, p. 165-193, jan. 1990.

SCHALK, H.J. and UNTIED, G., Wachstum und Arbeitslosigkeit. Gutachtenfür die Friederich Ebert Stiftung. Bonn. http://www.gefra-muenster.de (2000).

SOUZA JR, José Ronaldo de Castro e CAETANO, Sidney Martins. Produto Potencial como Ferramenta de Análise da Politica Monetária e da Capacidade de Crescimento da Economia Brasileira. Instituto de Pesquisa Econômica Aplicada (IPEA): Rio de Janeiro, outubro, 41p. 2013. Texto para Discussão 1881. 
